\title{
APROXIMACIÓN A LA MEDICIÓN DEL CAPITAL SOCIAL: UNA REVISIÓN LITERARIA
}

Martha Alicia Yánez Contreras Universidad de Cartagena Colombia

Amaury Jiménez Martínez Universidad de Cartagena Colombia 
Panorama Económico, Vol. 27 - No. 3 (Julio - Septiembre de 2019), pp. 631-654

Martha Alicia Yánez Contreras

Amaury Jiménez Martínez

\section{Aproximación a la medición del capital social: una revisión literaria}

\section{Resumen}

El artículo presenta un recorrido cronológico desde la década de los 90 s hasta el año 2015, resaltando los exponentes principales del capital social y las distintas metodologías desarrolladas para aproximarse a la medición del mismo, como un aporte a conocer los factores que determinan su desarrollo para conocer los aspectos fundamentales de estudio en los grupos de interés que se ven afectadas por fenómenos económicos, sociales, culturales, políticos y/o ambientales. Se resaltan los indicadores, variables proxies y fuentes de información primarias y secundarias utilizadas para medir el capital social. Se concluye que no hay una técnica de medición de capital social consolidada, y que la metodología varía de acuerdo al objetivo de la investigación, conceptualización, grupo de interés e información disponible.

Palabras clave: Capital social, medición de capital social, revisión literaria, indicadores de capital social, precursores de capital social, redes personales.

\section{Approximation de la mesure du capital social: revue littéraire}

\section{Résumé:}

L'article présente un parcours chronologique des années 90 à 2015, mettant en évidence les principaux représentants du capital social et les différentes méthodologies développées pour se rapprocher de sa mesure, comme une contribution à connaître les facteurs qui déterminent son développement pour connaître les aspects fondamentaux de l'étude dans l'intérêt les groupes affectés par des phénomènes économiques, sociaux, culturels, politiques et / ou environnementaux. Les indicateurs, les variables indirectes et les sources d'information primaires et secondaires utilisées pour mesurer le capital social sont mis en évidence. Il est conclu qu'il n'y a pas de technique pour mesurer le capital social consolidé et que la méthodologie varie en fonction de l'objectif de la recherche, de la conceptualisation, du groupe d'intérêt et des informations disponibles.

Mots clés: Capital social, mesure du capital social, revue littéraire, indicateurs du capital social, précurseurs du capital social, réseaux personnels.

\section{Approximation to the measurement of social capital: a literature review}

\section{Résumé:}

The article presents a chronological route from the 90 s to 2015 , highlighting the main exponents of social capital and the different methodologies developed to approximate its measurement, as a contribution to know the factors that determine its development to know the fundamental aspects of study in the interest groups that are affected by economic, social, cultural, political and / or environmental phenomena. The indicators, proxies variables and primary and secondary sources of information used to measure social capital are highlighted. It is concluded that there is no technique for measuring consolidated social capital, and that the methodology varies according to the objective of the research, conceptualization, interest group and available information.

Mots clés: Social capital, measurement of social capital, literary review, indicators of social capital, precursors of social capital, personal networks. 


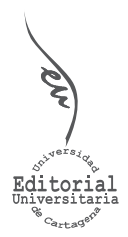

Artículo de Investigación

\section{Aproximación a la medición del capital social: una revisión literaria}

INFORMACIÓN DEL ARTíCULO

Recepción del artículo: 19/05/2019

Concepto de evaluación: 22/06/2019

Aceptación del artículo: 30/06/2019
Martha Alicia Yánez Contreras
Universidad de Cartagena, Colombia

Amaury Jiménez Martínez* Universidad de Cartagena, Colombia

\section{INTRODUCCIÓN}

Las comunidades están conformadas por individuos que tienen ciertos comportamientos y que se comunican con otros individuos con un objetivo en especial. Las relaciones, interacciones y dinámicas entre los miembros de una comunidad, así como también, la capacidad para resolver problemas y cambios que se presenten en la estructura social y la interacción misma, se conoce con el nombre de capital social (Gittel y Vidal, 1998; Saegert, Thompson, y Warren, 2001; Chaskin, Goerge, Skyles \& Guiltinan, 2006). Las medidas de la dimensión estructural del capital social, han hecho hincapié en el patrón de las conexiones entre un individuo y su red de conocidos, mientras que las medidas de la dimensión relacional de capital social enfatizan la naturaleza y la calidad de las interacciones y relaciones. En cambio, las medidas de la dimensión cognitiva de capital social se centran en las representaciones, las metas, las normas, los valores y la reciprocidad compartidos (Acquaah, Amoako-Gyampah \& Nyathi, 2014).

La importancia de medir el capital social radica en contribuir al bienestar de la comunidad mejorando, por ejemplo, los resultados de políticas en torno a le salud, economía, prevención del crimen, desempeño democrático e institucional. Autores como Brown \& Ashman (1996), Fox (1994) y Tendler \& Freedheim (1994), consideran el capital social como un activo para el desarrollo económico y político, que puede ser creado a corto plazo (Hiernaux Espinosa, 2013). Medir el capital social permite conocer cuáles son los factores que determinan el desarrollo del mismo y por ende saber a qué aspectos hay que apuntarle para su mejora.

\footnotetext{
*Autor de correspondencia: ajimenezm2@unicartagena.edu.co
} 
El capital social ha sido descrito y medido con tantos elementos tales que muchos investigadores han abogado por la separación de los mismos de acuerdo a las fuentes o los determinantes de que arrojan los resultados. Woolcock (1998) declaró que el capital social debe ser identificado por su origen o determinantes (por ejemplo, lazos sociales o redes sociales) en lugar de sus efectos o resultados (por ejemplo, recursos). Laursen, Masciarelli, \& Prencipe (2007) señaló que hay varios problemas en la medición del capital social, debido a la falta de una clara distinción entre las fuentes (o determinantes) y las consecuencias (o resultados) de capital social. Por ejemplo, Narayan \& Cassidy (2001) sostienen que algunos de las proxies que se han utilizado para medir el capital social, tales como la participación política, la seguridad, y la cohesión social son en realidad los resultados de capital social. En los estudios empíricos, se han aplicado principalmente, las medidas cuantitativas de capital social. Por ejemplo, muchos investigadores se han basado en encuestas dando como resultados índices de capital social, basados en organizaciones internacionales como el Banco Mundial y la Organización para la Cooperación y el Desarrollo. Los enfoques específicos para medir el capital social se han ido desde el simple uso de un indicador (por ejemplo, redes sociales) hasta el uso de varios indicadores (Acquaah, Amoako-Gyampah, \& Nyathi, 2014).

Este artículo tiene como objetivo dar a conocer cronológicamente cuales han sido las técnicas y/o instrumentos para medir el capital social a través de una revisión literaria. Para cumplir con dicho objetivo se consultaron las siguientes bases de datos: Dialnet, Ebsco Host, e-libro, Master File Premier, Science Direct, Scopus, Wiley, Academic Search Complete, Fuente Académica, Springer Link, Jstor, Scielo, Econlit, Pearson, McGraw Hill, Regional Business News, Business Source Complete, Biblioteca Virtual Colombiana, Redalyc, las cuales abarcan temas enmarcados en las ciencias económicas. Las palabras claves utilizadas durante la búsqueda, en inglés y español, fueron las siguientes: capital social, técnicas capital social, metodología capital social, medición capital social, valoración capital social, evaluación capital social, measurement of social capital, methodology of social capital, valuation of social capital; ya sea que se encontraran en el título, resumen y/o en el desarrollo del texto.

A su vez se encuentra divido en seis apartados incluyendo la introducción. Le sigue las bases para el capital social, donde se expone la conceptualización del mismo por algunos de sus exponentes principales. Luego, se describen las encuestas realizadas por países y entidades internacionales. Como cuarta parte, se encuentra la búsqueda de la medición del capital social en la última década del siglo XX. En el quinto apartado, se evidencia el auge por medir el capital social en los últimos años. Por último, las respectivas conclusiones, donde se resalta la falta de una medida estándar para cuantificar el capital social, evidenciando las continuas intenciones por parte de los autores alrededor del mundo por encontrar una forma para alcanzar la medición del mismo.

\section{BASES PARA EL CAPITAL SOCIAL}

En este apartado se contextualiza sobre la concepción del capital social, desde el punto de vista de varios autores pioneros en el tema, entre ellos, Pierre Bourdieu (1985, 2001), principal exponente de la sociología contemporánea, logra diferenciar tres tipos de capital: económico, cultural y social. Este último lo define como los recursos estructurales de un activo de capital para un individuo, que le facilita comportamientos dentro de un grupo social, en otras palabras, se refiere a las relaciones que se basan en el intercambio material o simbólico con otros individuos que contribuyen al mantenimiento de las mismas; lo que infiere que el volumen de capital social de un individuo dependerá de 
la amplitud de la red de conexiones que pueda movilizar y del volumen de capital que tengan los demás individuos de su red de relaciones.

A su vez, para Coleman (1988), que junto con Bourdieu fueron los pioneros en dar una definición detallada sobre el capital social, lo define como

El conjunto de fenómenos que implican la existencia de una particular estructura social, determinante para ciertos modos de actuar, tanto al nivel individual como colectivo. El capital social es productivo, y hace posible el logro de ciertos fines que no podrían ser alcanzados en su ausencia. Coleman da a conocer la estructura, las normas sociales, el concepto de lo individual y lo colectivo en pos de una productividad (Rojas Lopez \& Marin, 2006, p. 120).

Por su parte, Fukuyama (1999) define el capital social como "una norma informal que promueve la cooperación entre dos o más individuos (...) la vitalidad del capital social es esencial para el funcionamiento del mercado y la democracia". Asimismo, Putnam (1995), lo define como "los elementos de la organización social, tales como la confianza, normas y redes que establecen relaciones de reciprocidad activadas por una confianza social que emerge de dos fuentes, las normas de reciprocidad y las redes de compromiso ciudadano".

El concepto de capital social se ha expandido en cuanto su uso en los últimos 15 años de gran manera, como se evidenciará en los siguientes apartados. Coleman (1988), por ejemplo, define tres formas del capital, las normas sociales, los canales de información, y las obligaciones y expectativas que operan a través de redes relacionales que facilitadas por colectivos. Los individuos dentro de la estructura de las relaciones tienen la capacidad, en virtud de estas conexiones, para lograr fines que de otra manera no son posibles (1988, 1990). Putnam $(1993,2000)$ se centra en la confianza, normas y redes, y destaca el papel central de la participación ciudadana en una amplia gama de asociaciones que pueden facilitar la acción colectiva (Chaskin, Goerge, Skyles, \& Guiltinan, 2006, p. 491)

Ahora bien, existen autores que han distinguido distintos tipos de capital social, entre ellos: Burt (1992), Granovetter (1973), Coleman (1988) y Sampson (1999) que exploraron una distinción entre el capital social que es inherente en las relaciones internas, relaciones exclusivas (capital de unión) y externos, relaciones inclusivas (capital puente). Putnam (2000), Saegert, et al. (2001), Gittel \& Vidal (1998) y Warren (2001), estudiaron más a fondo el capital de unión o bonding capital, que promueve la solidaridad y la reciprocidad dentro de una comunidad o grupo; mientras que describieron que el capital de puente o bridging capital, proporciona acceso a los recursos y la información más allá del grupo. Del mismo modo, Woolcock (1998) se centra en las relaciones incrustadas (integración) y las relaciones autónomas (vinculación) en el nivel micro (integración social dentro y lazos sociales más allá de una comunidad o grupo, respectivamente) y sus contrapartes (integridad organizativa y de sinergia) en el nivel macro. Burt (2000; 2001) atribuye el capital social como una "metáfora acerca de la ventaja", que explica cómo las personas que están mejor conectadas pueden beneficiarse de esas conexiones. Se promueve la ventaja mediante la vinculación de los actores a los recursos a través de las relaciones dentro de las redes y, sobre todo, a través de agujeros estructurales, éstos son las brechas entre contactos no redundantes que vinculan a los individuos a conectarse con redes distintas de la suya; proporcionando acceso adicional a la información, los recursos y las oportunidades (Burt, 1992; Chaskin, et al., 2006, p.p. 491-492). 


\section{ENCUESTAS PARA MEDIR EL CAPITAL SOCIAL}

La forma más aceptable de manifestación del capital social es a través de la confianza, razón por la cual es utilizada recurrentemente como proxy para su medición por medio de las encuestas. Entre las encuestas se pueden distinguir dos grupos: aquellas diseñadas específicamente como instrumentos de medida del capital social y aquellas encuestas generales que incluyen la medición de alguna dimensión del capital social. En el primer grupo, el de las encuestas específicas, se pueden destacar el Instrumento de Medida del Capital Social del Banco Mundial (Social Capital Assessment Tool) y el Estudio de Referencia del Capital Social Comunitario (Social Capital Community Bechmarck) de la Escuela Pública de la Kennedy School. En el segundo grupo de encuestas genéricas se puede citar la Encuestas Social de Estados Unidos (General Social Survey), la Encuesta General de Familias de Inglaterra (General Household Survey), la Encuesta Mundial de Valores (World Values Survey) o la Encuesta Social Europea (Social European Survey) (García Montalvo \& Reynal Querol, 2003).

Algunos aspectos que tratan la mayoría de las encuestas son: confianza general y específica, densidad de las relaciones sociales, participación en asociaciones voluntarias, participación cívico-política, intensidad de las normas cívicas de cooperación y preguntas contextuales, sea: género, nivel educativo, edad, etnia, entre otros (García Montalvo \& Reynal Querol, 2003). Es así como varios países han desarrollado marcos para la medición de capital social como: Nueva Zelanda, Australia, Reino Unido, Canadá, Finlandia, Estados Unidos, Colombia -por medio del Barómetro de Capital Social BARCAS ${ }^{1}$ 2005 implementado por Sudarsky (2007), el cual ha sido aplicada en tres ocasiones (1997, 2005 y 2011) tomando como referencia la Encuesta Mundial de Valores (Foliaco Gamboa, 2013)-, en Ghana y Uganda -aplicada Narayan \& Cassidy (2001) -, entre otros países (Universidad del Pacífico, 2009). Asimismo, entidades como la Organización para la Cooperación y el Desarrollo Económicos (OCDE) y el Banco Mundial que buscan crear estándares estadísticos a nivel internacional (Parnanen \& Iisakka, 2006).

\section{NACIMIENTO DE UNA TENDENCIA: MEDIR EL CAPITAL SOCIAL (1990-2000)}

La primera publicación de Putnam, Leonardi, \& Nanetti (1993) fue el detonante del desarrollo del concepto de capital social y su medición, resaltando el legado de Coleman $(1988 ; 1990)$ con las relaciones de norte y sur de Italia, asumiendo el nivel de asociacionismo como un indicador válido del nivel de capital social, y Bourdieu (1985). En el experimento de Putnam, los altos valores del asociacionismo del norte de Italia contrastan con los niveles reducidos del sur, concluyendo que el capital social se presenta como un conjunto de redes sociales, normas informales y vida asociativa (Caballero Míguez \& Kingston, 2005).

Posteriormente, Hall (1999) en su ensayo sobre el Capital Social en Gran Bretaña basado en la construcción de las relaciones de confianza y reciprocidad mutua, estudia los siguientes aspectos basado en encuestas nacionales como aproximación a la medición del capital social: miembros en asociaciones voluntarias; obras de caridad; asociaciones informales; efectos de generación; y nivel de confianza social.

1. Aplicada por otros autores en los siguientes apartados del artículo. 
Narayan \& Pritchett (1999) implementan la Encuesta de Capital Social y Pobreza en abril y mayo de 1995 para 1376 hogares con el objetivo de recopilar datos sobre la participación en asociaciones y la confianza. La encuesta de capital social abarca tres dimensiones: 1) pertenencia a grupos; 2) características de los grupos a los que pertenece el hogar; y 3) valores y actitudes de la persona, en particular su definición y nivel de confianza expresado en varios grupos y percepción de la cohesión social. Posteriormente ingresan la información a un modelo econométrico de regresión lineal para ver el efecto del capital social en los ingresos.

\section{EVIDENCIA DEL AUGE DE LA MEDICIÓN DEL CAPITAL SOCIAL (2001-2015)}

En los últimos 15 años, se ha presentado un aumento considerable en las investigaciones dedicadas a medir el capital social ya sea por medio de encuestas de entidades gubernamentales, encuestas propias, entrevistas, modelos econométricos, estudios de casos, entre otros. Muestra de ello son los siguientes trabajos encontrados durante la revisión literaria, presentado de manera cronológica.

En el nuevo milenio, Durston (2001) en aras de evaluar el capital social en comunidades campesinas en Chile, utiliza la Metodología de Evaluación Rápida del Capital Social (MERCS) la cual implementa los siguientes instrumentos: la aspiradora etnográfica, que registra todos los datos posibles sobre una gama de temas; elaboración de mapas catastrales de recursos naturales; elaboración de genealogías; el enfoque dramático, que consiste en el registro minucioso de eventos significantes para las personas.; el registro de relatos (historias de personas en organizaciones); la línea del tiempo (cambios significativos en el pasado de la comunidad); líneas de tendencia (cómo la gente percibe los cambios que se han dado en el tiempo); sociograma (tabla de doble entrada donde se sitúan a los hogares que integran una localidad); encuesta socioeconómica; análisis organizacional/institucional (diagrama de Venn); y la matriz de análisis de conflicto (niveles de ocurrencia de los conflictos, modalidad de resolución).

Esta metodología se concentró en la recolección de información, su ordenamiento y análisis, pero desarrolló poco el proceso de estructurar ese análisis en términos de una evaluación. Se hizo necesario experimentar con la metodología de grounded theory como un posible camino para estructurar esa evaluación en forma ordenada y rigurosa:

La teoría aterrizada (o teoría fundamentada) es una metodología que busca generar hipótesis de trabajo nuevas y más cercanas a la realidad empírica. Se basa en trabajo de campo y pretende dar un carácter científico al trabajo etnográfico. Algunos aspectos para destacar de la metodología son: comparación de casos similares (para buscar nuevas variables); reflexiones teóricas sobre los datos para generar nuevas preguntas; debates e intercambios dialécticos; entrevistas continuas hasta que se encuentre redundancia de información; codificación ${ }^{2}$ y

2. Tipo de codificación: a) Abierta: la primera codificación a realizar de los textos es sustantiva y descriptiva. Se busca máxima la exhaustividad en el detalle. Se divide en dos subtipos: In vivo, términos usados por los entrevistados y de observación, por los investigadores. b) Axial: Busca la detección de relaciones entre códigos, para la generación de categorías más abstractas. c) Selectiva: Postula relaciones conceptuales entre las categorías, para llegar a categorías centrales y a una sola 'categoría raíz': en torno a la cual se configura una red conceptual. (Durston, 2001, p.p. 7-8) 
categorización de información recolectada; interpretaciones del investigadores; y por último, la formulación de hipótesis de trabajo (Durston, 2001, p. 8).

Para el mismo año, Grootaert (2001) realiza un estudio de las instituciones locales en tres países (Bolivia, Burkina Faso e Indonesia) donde tuvo como objetivo investigar el papel del capital social y las instituciones locales en el bienestar y la pobreza. Los datos fueron recolectados a nivel de comunidad, barrio y hogar; para ellos se utilizaron tres instrumentos: a) la información sobre los servicios comunitarios se obtuvo a través de entrevistas con los principales informantes, como el jefe de la aldea, maestros, proveedores de salud, entre otros, que a su vez, fue complementada con información sobre la economía local (infraestructura y distancia a los mercados), la sociedad local (etnia y religión) e instituciones locales; b) los servicios comunitarios también se discutieron con grupos de hogares para conocer la perspectiva de la comunidad sobre la calidad del servicio, su experiencia con la acción colectiva y puntos de vista sobre las instituciones locales y proyectos de desarrollo; c) para las instituciones locales más importantes, se llevaron a cabo entrevistas con los líderes, miembros y no miembros, con el fin de obtener una visión equilibrada del papel de las instituciones en el pueblo, su evolución en el tiempo, actividades principales, relaciones con otras instituciones y el gobierno, fortalezas y debilidades.

La parte crítica fue la recolección de datos por medio de una encuesta de hogares que tuvo como objetivo captar la participación real de los hogares en las instituciones locales, uso de servicios e información que identificara el nivel de bienestar de los hogares; dicha encuesta consta de seis secciones: información demográfica sobre los miembros del hogar; participación en las instituciones locales; características de grupos más importantes; perfiles de prestación de servicios; percepciones de confianza de la comunidad y la colaboración; y economía familiar y estrategias de afrontamiento.

Por otro lado, para Arriagada (2003) la acción participativa se considera como la metodología que permite captar la participación de los actores sociales en lo grupos informales. La cual se complementa con encuestas sociales sobre valores y confianza. Asimismo, se evalúa la ausencia de capital social por medio de tasas de criminalidad, uso de drogas, suicidios, entre otros (Fukuyama, La gran ruptura. Naturaleza humana y reconstrucción del orden social, 2000). Resaltando que:

Existen serias dificultades en la medición del capital social. Se sostiene que si bien es posible medirlo, sólo por casualidad esas mediciones cumplirán con los requerimientos básicos de rigor científico (Fine, 2001). Sin duda, que la diversidad de definiciones existentes se expresa también en diversidad de formas de medición (Arriagada, 2003, p. 22).

Ahora bien, Novakovsky (2003) para medir el capital social recomienda tener en cuenta el nivel macro social, por medio de encuestas de organizaciones como la CEPAL, el índice de capital humano, encuestas de opinión, de hogares, ente otras; así como también, la construcción de indicadores indirectos y criterios socio-institucionales como la existencia de acuerdos, expectativas, compromisos y reglas, participación activa de las organizaciones, líderes comunitarios y cultura institucional. Por su parte, Van Der Gaag \& Snijders (2003), resaltan tres métodos para la medición de capital social: basados en nociones de volumen/extensidad del capital social, diversidad en las relaciones y presencia de recursos de capital. 
A si mismo, Ayerbe Echeberría y otros (2005) afirman que para medir el capital social, se deben realizar tres actividades: la detección de las redes organizacionales internas y externas; construcción de los indicadores que midan el capital social; y el proceso de medición. Dentro de la medición del capital social se evalúan dos tipos de capital social: el capital social comunitario y el capital social individual. En ambas tipos de capital se miden tres conceptos: socialización (nivel integración), legitimidad (confianza) y la participación (la interacción real). Utilizaron un instrumento de medición ad hoc para la medición del capital social. Primero, se aplicó como técnicas cualitativas el juicio de expertos, el análisis de datos secundarios y las dinámicas de grupo. Posteriormente se aplicó las técnicas de análisis factorial y la correlación entre las variables (y dimensiones explicadas en el artículo de los mismos autores mencionados con anterioridad), como aspecto cuantitativo; metodología también utilizada por Narayan \& Cassidy (2001).

Para el año 2006, Rojas \& Marin, en su estudio de aproximaciones a la medición del capital social desde la confianza, tienen dicho factor como una variable cualitativa clave para la construcción del capital social en la Facultad de Minas de la Universidad Nacional de Colombia. La confianza como variable, tuvo en cuenta los conceptos de relaciones fraternales como amistad y experiencias previas; conceptos basados en valores que forjen la credibilidad en la persona como la responsabilidad, puntualidad y cumplimiento. En la construcción del capital social, se diseñó una encuesta para observar la relación de confianza entre compañeros, estudiantes-profesores y alumnos en la institución. El estudio se basó en la correlación entre las variables, estableciendo un muestreo estratificado, asignando preguntas con opción de respuesta cerrada (siempre, casi siempre, a veces, casi nunca, nunca) y estableciendo un porcentaje a cada respuesta de la encuesta: 0\% nunca, 10\% casi nunca, 50\% a veces, $80 \%$ casi siempre y $100 \%$ siempre. Con lo anterior, la confianza se calcula con base a cada variable (pregunta) que corresponde a la suma aritmética de los porcentajes para cada categoría y se asigna el mismo peso a cada una de ellas. Posteriormente se calcula la confianza promedio de los actores (compañeros, profesores, norma institucional, etc); luego la confianza promedio para cada estrato utilizando la media aritmética y finalmente se halla la confianza en la facultad con el promedio ponderado fundamentado en el peso de cada estrato.

Algunos autores como Serrano, Alarcón \& Tassara (2006) crearon un índice de medición de capital social, para el cual se definieron un conjunto de dimensiones: a) información, que se refiere al manejo de conocimiento de los miembros de la sociedad respecto a un tema de especial; b) participación, acción voluntaria y no renumerada de personas que se unen a organizaciones con un objetivo en común; c) redes, vínculos de los miembros de la comunidad con otros miembros o grupos o instituciones; d) confianza; e) empoderamiento y liderazgo; f) ciudadanía y cultura cívica; g) acción colectiva para la provisión de servicios/ recursos. Una vez definida las dimensiones se procedió a crear una encuesta y una prueba piloto del instrumento para su validación. Las dimensiones consideradas en el instrumento (encuesta) fueron: grupos y redes; confianza y cohesión social; acción colectiva y cooperación; información; empoderamiento y acción política.

Michell \& Bosset (2007) afirman que tanto la pertenencia a las organizaciones, es decir, la densidad de miembros y la confianza social (confianza general en las personas) son indicadores para medir el capital social estructural y cognitivo, respectivamente. Se suele afirmar que el capital social estructural incluye tanto los lazos de unión (relaciones de cooperación entre los miembros de una red social que comparten un sentido de identidad social) y los lazos puente (relaciones de cooperación entre las personas que son socio-demográfica y / o económicamente) (Szreter \& Woolcock, 2004). El capital social 
se midió a través del Cuestionario de Capital Social Integrado (SC-IQ) desarrollado por el Banco Mundial. Como instrumento estadístico usaron dos conjuntos de análisis factorial exploratorio para examinar las dimensiones del capital social. Las estructuras factoriales (cognitiva y estructural) se evaluaron individualmente y luego se compararon. Mientras que el análisis factorial es útil en la comprensión dimensional del capital social, las regresiones multivariables exploraron las relaciones del capital social, con resultados hipotéticos. Las regresiones fueron ponderados por el tamaño del hogar, con los datos agrupados por comunidad utilizando el "svyreg o svyologit" en Stata.

Enel mismoaño, Valdivieso(2007)para medir el capital social tieneen cuenta tres variables que son: la participación convencional, no convencional o comunitaria, y la confianza en las relaciones interpersonales e instituciones. A su vez tienen en cuenta variables socioeconómicas y actitudinales como: sexo, nivel educacional, nivel de ingreso, apoyo a democracia, participación política, confianza en otra gente, instituciones, entre otros. Por medio de modelos de regresión lineal múltiple y el método backward selection para descartar las variables que no sean significativas, se observaron las relaciones causales, seleccionando variables del capital sociales en calidad de variables dependientes. Para el caso de las variables dependientes binarias (participación en asociaciones religiosas y discute sobre la política) se realizó una regresión logística.

Posteriormente, la Fundación BBVA con el direccionamiento de Pérez García, F., Serrano Martínez, L. \& Fernández de Guevara Radoselovics (2008) establecen dos metodologías de la medición de capital social: un modelo teórico y uno práctico. El primero, tomando como base en Pérez García, Serrano Martínez, Montesinos Santalucía, \& Fernández de Guevara Radoselovic (2006) consideran que las relaciones económicas son el pilar para la generación del capital social, considerando al mismo como cualquier otro tipo de activo, según la función de rentabilidad futura esperada. Para ello, como lo afirman Glaeser, Laibson, \& Sacerdote (2002) y siguiendo la metodología para medir el capital físico de la OCDE (2001) se realiza un modelo de decisión optima donde el agente económico invierte en capital social si los rendimientos futuros son mayores a sus costos. Por otro lado, el modelo práctico se realiza por medio de encuestas donde se tienen las siguientes variables como proxies del capital social: a) grado de conexión de la red social (créditos/ producto interno bruto); b) coste marginal de la inversión en capital social (proporción de población en edad de trabajar con mínimo estudios medios); c) tasa de depreciación (tasa de desempleo) y tasa de supervivencia del capital social (grado de cumplimiento de las obligaciones económicas); d) dimensión de la red social (número de ocupados); e) índice de desigualdad de la renta (índice de Gini); f) horizonte temporal de pertenencia a la sociedad (esperanza de vida); g) tasa de descuento temporal (4\%) y grado de reciprocidad en la sociedad (valor arbitrario); y h) población total de cada población de estudio.

Siguiendo la idea del grupo de autores anteriores, donde se plantean dos modelos y uno de ellos práctico, Sabatini (2009) resalta que el punto de partida es igualmente un análisis empírico, el cual se realiza mediante el reconocimiento de la multidimensionalidad del concepto de capital social, que no puede ser representado por un solo indicador. El estudio se basa en aproximadamente 200 indicadores de cuatro principales dimensiones: fuertes lazos familiares, débil lazos informales; organizaciones de voluntarios y la participación política. Los datos son establecidos en forma conjunta de las múltiples encuestas llevadas a cabo por el Instituto Nacional Italiano de Estadísticas (ISTAT), sobre una muestra de 20000 hogares entre 1998 y 2002. 
Cheung \& Kwok-hong (2010) encontraron una manera de aclarar la medición del capital social por medio de la diferenciación de dos enfoques: oportunidades e intercambio. El capital social basado en oportunidad incorpora la participación de la organización, las redes sociales (densidad, homogeneidad y proximidad), la confianza, continuidad de la relación, confianza generalizada, la ayuda recibida, mientras que el capital social basado en el intercambio consiste en la inversión y la reciprocidad de ayuda. Un indicio empírico para verificar las mediciones de capital social es su previsibilidad moral. Con base en datos de la encuesta de 201 residentes japoneses, el estudio examinó diversas mediciones de capital social basado en 10 indicadores (mencionadas anteriormente) y su predicción de la moral. A su vez, se realizaron correlaciones parciales y efectos estandarizados para cada indicador. Los resultados revelaron que el capital social basado en el intercambio fue suficiente para contribuir a la moral, y esencialmente representa la parte saludable del capital social. Como el capital social basado en la oportunidad no tenía ninguna contribución a la moral, una medición refinada de capital social solo puede concentrarse en su base en el intercambio.

A su vez, se desarrollaron medición netamentes cuantitativas como el uso de ecuaciones estructurales de Fornoni, Arribas, \& Vila (2011), que se estima y se valida a partir de una base de datos que incluye información de 282 empresarios argentinos que respondieron a un cuestionario. El modelo de medición considera las dimensiones propuestas por Koka y Prescott (2002), es decir, relacionales, de recursos y estructurales. Por otro lado, el modelo de medición propuesto por Batjargal (2003) integra tres dimensiones del capital social: la primera dimensión depende de la estructura de la red y las propiedades de la posición ocupada por el agente en la red (dimensión estructural), la segunda dimensión resume las características de las relaciones del agente, como la confianza, la longevidad del enlace, etc. (dimensión relacional) y la tercera dimensión mide el valor de los recursos que los agentes conectados en red son capaces de proporcionar los recursos (dimensión). Estas tres dimensiones del capital social no son directamente observables y necesitan ser medida indirectamente a través de una serie de indicadores observables. Varios de estos indicadores se han propuesto en la literatura. Por ejemplo, Nahapiet \& Ghoshal (Social capital, intellectual capital, and the organizational advantage, 1998) sugieren los siguientes indicadores de la dimensión estructural de capital social: número de enlaces y otras características de la configuración de la red, la densidad, jerarquía o nivel de conectividad. A su vez, para medir la dimensión relacional, Koka y Prescott (2002) sugieren los siguientes indicadores: múltiples enlaces que representan la existencia de diferentes relaciones simultáneas entre dos agentes y enlaces repetidos que representan el número de relaciones que dos agentes mantienen en el tiempo. Un indicador de confianza alternativa puede ser la duración de la relación (Gulati, 1995).

Rangel J. \& Saíz V. (2011) hacen una aproximación a la medición y análisis de capital social basado en las relaciones (usuario-empresa) por medio de un análisis estadístico multivariado.

"La confianza se entiende como el grado de seguridad o convicción de que han de cumplirse un con $\neg$ junto de expectativas de manera exitosa, en un contexto específico y durante un periodo de tiempo determinado. Básicamente, se trata de la no existencia de la sensación de incertidumbre ante las acciones de las personas o las instituciones, lo que hace factible asumir un grado de predictibilidad en las acciones sociales" (Rangel J. \& Saíz V., 2011, p. 83). 
Dicha confianza se mide desde cada integrante de la comunidad sea: vecinos, asociaciones comunales, fuerzas militares, iglesia, etc.; así como también por rangos de edad, estrato, barrio, género. Creando un índice de gestión social que mida las dimensiones de confianza (medida en un rango de 1 a 5 , siendo 1 la calificación más baja y 5 la más alta), apoyo (cooperación, medida igual que la anterior), participación (solidaridad) e interés valoradas según su existencia a través de un criterio dicotómico de "Si" o "No". Utilizando el análisis de correspondencias múltiples, asignando ponderaciones a cada una de las categorías mencionadas anteriormente. Luego se determina un valor para cada individuo (como la suma de las ponderaciones de sus categorías). Una vez establecido los promedios del IGS (Índice de Gestión Social Empresarial) por barrio (o localidad) se decide establecer el factor del indicador entre cero y uno, siendo cero los grupos de usuarios que deben trabajar para mejorar la imagen de la empresa y vincularlos en redes sociales que generen bienestar a la comunidad. Y siendo uno usuarios que tienen buena imagen de la empresa y son más participativos en las actividades de la misma, generando la formación de redes sociales.

Otro estudio donde se toma como muestra una comunidad, es el de González Ordóñez (2011) que con el objeto de analizar el capital social existente en la comunidad ubicada en el sector Guayapa, se siguió del procedimiento de la teoría fundamentada implementado por Strauss \& Corbin (Bases de la investigación cualitativa. Técnicas y procedimientos para desarrollar la teoría fundamentada, 2002). Las unidades de análisis fueron las organizaciones comunitarias asentadas en el sector. Los informantes fueron dos integrantes de cada organización comunitaria y para la selección se utilizó un muestreo intencional donde se tenía en cuenta que pertenecieran y participaran en una organización comunitaria, que tuvieran tiempo suficiente trabajando en dichas organizaciones, además de que contaran con conocimientos sobre la participación y organización comunitaria, que vivieran en la comunidad y que tuviesen disposición para suministrar la información. Para la recolección de la información se aplicaron técnicas como la observación participante y entrevistas no estructuradas. Por su parte, el análisis de datos se realizó mediante la codificación abierta, axial y selectiva. Teniendo en cuenta aspectos como: instituciones, reglas y normas; redes de capital social; elementos culturales y relaciones sociales.

Desde la perspectiva de la presencia de capital social a nivel empresarial en una cadena productiva, se trae a colación el estudio de Piña, Castellanos \& Morales (2011), el cual, en aras de cuantificar y cualificar la presencia de capital social en la cadena aloe del estado Falcón, se basaron en el análisis de caso con el objetivo de caracterizar la naturaleza del capital social de las asociaciones de productores presentes en la cadena aloe. Los sujetos de estudio fueron la junta directiva de asociaciones y cooperativas de productores y de plantas de procesadores de aloe. Por su parte, el procedimiento de investigación fue por medio de una conversación semiestructurada a partir de un cuestionario base. Las variables estudiadas fueron: información y comunicación (medios de difusión de información en la asociación; frecuencia del flujo de información; satisfacción con el manejo de información), confianza y solidaridad (confianza en los miembros de la asociación; relaciones personales con vecinos; ambiente de cordialidad y solidaridad), acción colectiva (promoción de proyectos dentro de la asociación; participación, como asociación, en actividades comunales; orientación política de la asociación), y por último, grupos y redes (motivación para pertenecer a la asociación, papel de la asociación para resolver problemas; participación como miembro de la asociación; membresía en otros grupos y redes). Evidenciando la presencia de una fuente capital social materializado en la actitud y proactividad de los miembros de la cadena. 
Ahora bien, en el informe de resultados de la medición de capital social en Cartagena (Colombia), llevado a cabo por el equipo de investigación Hurtado, García \& Copete (2012) evidencia el uso del instrumento Barómetro de Capital Social (BARCAS). El BARCAS busca realizar una caracterización de la sociedad colombiana en términos de indicadores del capital social y actividades políticas. Se usaron métodos estadísticos multivariados como el análisis factorial para disminuir los datos y definir grupos de variables para resumir aspectos comunes. Las dimensiones tratadas fueron: solidaridad y mutualidad, relaciones horizontales, jerarquía o articulación vertical, confianza institucional, control social, participación cívica, participación política, información y transparencia, republicanismo cívico, medios.

Por su parte, las economistas españolas Portela \& Neira (2012) plantean una aplicación econométrica para tratar de cuantificar si las variables del capital social elegidas, tienen influencia en el crecimiento económico. Resaltan que existen diferentes indicadores para medir el capital social, sea de manera cuantitativa o cualitativa, pero que no hay ninguno que tenga como resultado una valoración directa dado de que no existe un mercado para el capital social por su característica de bien público hace complicado su valoración.

La confianza permite la cooperación sin la influencia directa del poder o del mercado, lo cual coloca a esta variable en un lugar importante en la construcción y el mantenimiento del orden social y, por consiguiente, del capital social. El grado de confianza interpersonal viene medido por el porcentaje de personas que responden que se puede ser confiado cuando se plantea la pregunta: Generalmente hablando, ¿cree usted que la mayoría de la gente puede ser confiada o que hay que tener cuidado en el trato con la gente? (Portela \& Neira, 2012, p. 192).

Alcaide Lozano (2013) con respecto al capital social y su medición en perspectiva atributiva y reticular usó variables provenientes de la Encuesta de Calidad de Vida en el Trabajo para 2008. Las dimensiones e indicadores del capital social en la Encuesta de Calidad de Vida en el Trabajo son las siguientes: confianza (baja/media/alta) en los superiores, compañeros y subordinados; satisfacción en el puesto de trabajo actual (Baja/ media/alta); calidad de las relaciones (malas/ni buenas ni malas/muy buenas) entre los directivos y empleados y entre los trabajadores; participación en asociaciones y sindicatos; e influencia (poca/ni mucho ni poco/mucho) para el cambio de trabajo actual. A partir de las variables expuestas se observan los niveles de capital social en tanto qué relaciones son más fuertes o más débiles.

Así mismo los siguientes autores propusieron una metodología desde el manejo y estudio de redes, ellos fueron: Buciega \& Esparcia (2013), que para medir el capital social, llevaron a cabo una aproximación potencial del análisis de redes (ARS) para analizar la presencia del mismo. Se basó en cuestionarios estructurados que se cumplieron mediante entrevistas personales a los miembros implicados. Teniendo en cuenta una dimensión interna que toma como referencia el capital social que se genera en el grupo de miembros de estudio se analizan las relaciones existentes. Partiendo de una frecuencia e intensidad elevada de las relacione sacaba generando más confianza, cohesión, y compromiso.

Palafox Moyers, Espejel Blanco, Burgos Flores, \& García Pequeño (2013) desarrollaron una metodología cualitativa y cuantitativa. La primera, consistió en la elaboración de entrevistas. La segunda, se utilizó las técnicas multivalentes de Análisis de Componentes Principales y Modelos Estructurales PLS con el objetivo de hallar las relaciones causales entre las variables escogidas por medio de mínimos cuadrados ordinarios. Las principales 
variables fueron: confianza y solidaridad; acción colectiva y cooperación; cohesión e inclusión social. Posteriormente se usó el cuestionario elaborado por el Banco Mundial para determinar el capital social.

Para el año 2014, relucieron una vez más, los estudios cuantitativos (econométricos, análisis factoriales, estudios psicométricos, etc.), uno de ellos fue el aportado por Acquaah, Amoako-Gyampah, \& Nyathi (2014), los cuales en su investigación sugieren que la medición del capital social refleja una naturaleza multidimensional, y los diversos componentes se podría resumir en cuatro grandes categorías: redes, relaciones y conexiones; confianza; compromiso cívico y actividades voluntarias (incluida la cooperación, la participación política, la participación social, asociativa membresías, voluntariado comunitario, etc.); y normas cívicas, normas y valores compartidos.

Chung, Choi \& Seung-yoon (2014) basados en encuestas a nivel internacional llevadas a cabo por el Banco Mundial y la Organización para la Cooperación y Desarrollo Económico, seleccionaron algunas preguntas que reflejaran el contexto de la sociedad coreana. Con el fin de investigar los patrones de las dimensiones del capital social como la participación social, la confianza, normas y redes, se ejecutó el análisis factorial exploratorio. Teniendo en cuenta el número de factores que se determina en función del valor propio, porcentaje acumulado, gráfico de sedimentación e indicador de Tucker-Lweis. Este último se produce utilizando el método de máxima verosimilitud. Posteriormente con el resultado del análisis factorial, se regresa a las preguntas asociadas con la sociedad coreana y se calibran los atributos conceptuales (dimensiones) del capital social por medio del enfoque fuzzy-set.

Fernández Niño, Pinzón Flórez, Moreno Montoya, \& Idrovo Velandia (2014), presentan una adaptación al español y validación psicométrica de una escala para la medición de capital social en contextos rurales, también conocida como la escala de capital social cognitivo de Wang y otros autores (The flip-side of social capital: The distinctive influences of trust and mistrust on health in rural China, 2009). Aplicando 1200 cuestionarios a adultos en 12 veredas de Tierralata (Colombia) por medio de un muestreo aleatorio simple estratificado a partir de la base de datos del Sistema de Identificación de Beneficiarios (SISBEN), y un análisis factorial exploratorio a partir de una matriz de correlación policórica. Para el respectivo análisis se sugiere la existencia de dos factores principales distribuidos así: 7 ítems para el factor 1 (confianza) (valor propio 3.23) y 2 ítems para el factor 2 (desconfianza) (valor propio 1.40). La confianza y desconfianza se miden mediante 10 preguntas (tipo Likert de cinco puntos, desde muy en desacuerdo a totalmente de acuerdo) sobre las actitudes hacia la interacción social en la comunidad. Posteriormente, en el análisis estadístico, las características de la población fueron descritas para cada vereda y las diferencias por vereda de las variables cualitativas fueron exploradas con la prueba ji-cuadrado usando el procedimiento de corrección de Marascuilo. Las medianas de las variables cuantitativas fueron comparadas por medio de las pruebas de Kruskal-Wallis y por último se realizó el análisis factorial por el método de extracción del factor componente principal. Cabe resaltar que todos los análisis fueron hechos en el programa estadístico Stata 11.

Portales Luis (2014) utilizó la técnica de redes personales como estrategia de recolección de información y un análisis de componentes principales categóricos para caracterizar el capital social de96hogares mexicanos en situación de pobreza. Desdela visión minimalista del concepto la cual considera el capital social como la suma de las relaciones sociales que presenta un determinado actor y las redes sociales que producen la interacción de 
estas relaciones. Para que este entendimiento pudiera ser aplicado a nivel de los hogares fue necesario identificar las relaciones sociales y las redes sociales que los diferentes miembros del hogar habían utilizado para tener algún tipo de beneficio. En el caso de esta investigación se tomó como beneficio el acceder a los diferentes componentes que conforman el capital económico y humano de los hogares. Se construyeron tres variables relacionadas con el capital social mismas que conforman las variables independientes del modelo estadístico utilizado. Las primeras dos variables se construyeron con base al grado de cercanía o lejanía de las relaciones sociales del hogar. La construcción de estas dos variables tuvo como base la generación de un indicador que dio muestra de la cercanía o lejanía de las relaciones sociales de los hogares en función del tipo de capital al que dan acceso. A su vez, construyó el Índice de Proximidad de Relaciones Sociales (IPRS) que muestra la cercanía o lejanía de las relaciones sociales de los hogares de acuerdo al tipo de capital al que tengan acceso. Dicho índice es un aproximado a la idea de Granovetter (1973) sobre lazos fuertes y débiles, así como de Putnam (1995) y Woolcock (2001) sobre los tipos de capital social: cierre, puente y enlace. El IPRS se creó por medio del Análisis de Componentes Principales, el cual se utiliza cuando las variables son categóricas. Una vez aplicado esa metodología, se pasa a aplicar el modelo factorial (Gadermann, Zumbo \& Guhn, 2012).

Ahora bien, Saz Gil \& Gómez Quintero (2015) hacen una aproximación a la cuantificación y caracterización del capital social en la provincia de Teruel, España. Usaron una gran variedad de fuentes secundarias ${ }^{3}$. Con respecto al análisis de confianza se estudió el nivel de confianza de los españoles en caso de necesitar ayuda; la confianza en el futuro de la situación económica personal en el municipio de estudio; la confianza en el futuro de la situación económica del municipio en comparación con el resto; la confianza en los representantes políticos (población que se abstuvo en las elecciones municipales); y la población con discapacidad según frecuencia con la que ha visto a alguno de sus familiares/amigos/vecinos (redes sociales y atención a la enfermedad). Posteriormente se hizo un análisis del nivel de asociacionismo teniendo en cuenta un análisis de las organizaciones de primer nivel por medio del número de organizaciones constituidas formalmente por cada 1000 habitantes; la distribución de organizaciones sociales por fecha de creación; y las organizaciones de segundo nivel entre activas e inactivas.

Por último, Zambrano, Duque \& Manzano (2015) en aras de medir el capital social en el barrio La Primavera del municipio de San José de Cúcuta, Colombia, se basa en Kliksberg (1999) el cual define el capital social como "normas y redes que facilitan la acción colectiva y contribuyen al beneficio común, considerando a los seres humanos como seres sociales que se encuentran inmersos en un entorno y no simplemente como unidades individuales que piensan en términos racionales" (Zambrano, Duqe \& Manzano, 2015), identifica cuatro dimensiones básicas del capital social: el clima de confianza al interior de una sociedad, la capacidad de asociatividad, la conciencia cívica y los valores éticos predominantes en una sociedad.

\footnotetext{
3. Barómetro del Centro de Investigaciones Sociológicas (CIS) de septiembre de 2010, $n^{\circ}$ 2844; índice de confianza del consumidor de 2011, realizado por el Instituto de Crédito Oficial; Barómetro de Opinión de Aragón realizado en la primavera de 2011; estadísticas electorales del Ministerio del Interior, a partir de cuyos registros el Instituto Aragonés de Estadística elaboró los datos de las votaciones; Encuesta sobre Discapacidades y Autonomía personal; estadísticas del sector no lucrativo en Aragón; bases de datos del Gobierno de Aragón de asociaciones, fundaciones y colegios profesionales, además de trabajos previos del grupo de investigación GESES (Grupo de Estudios Sociales y Económicos del Tercer Sector) de la Universidad de Zaragoza. A partir de los datos recabados se ha realizado un análisis descriptivo.
} 
Ahora bien, todos los autores mencionados anteriormente presentan una metodología implementada para medir el capital social de acuerdo al grupo de interés de cada investigación basándose en la conceptualización inicial que tengan sobre el capital social.

\section{Relaciones existentes entre el concepto de capital social y la metodología implementada}

Los autores principalmente se basan en concepciones de los principales exponentes: Bourdieu, Coleman, Putnam y Fukuyama. Es así como incluso Putnam junto con Leonardi \& Nanetti se base en Coleman $(1988,1990)$ gracias a la definición de capital social basado en la relación de las tradiciones cívicas, les permitió determinar el nivel de asociacionismo de las comunidades como próximo al capital social (Caballero Míguez \& Kingston, 2005).

Por otro lado, Durston (2001) para usar la Metodología de Evaluación Rápida del Capital Social (MERCS) se fundamentó en la teoría aterrizada o fundamentada (marco conceptual en el cual González Ordoñez (2001) también se basa) y en la conceptualización del capital social de una manera conductivista, dando un privilegio a las relaciones sociales y la conducta de las personas por encima de los aspectos normativos y culturales, tomado de los ideales de Coleman y Bourdieu, mencionados con anterioridad. A diferencia de North, Putnam y Fukuyama, que tienen en cuenta los aspectos culturales y de valores.

Grootaert (2001) se basa conceptualmente en Portes (1998, p. 6) el cual afirma que "el capital social representa la capacidad de los actores para asegurar beneficios en virtud de la membresía en las redes sociales u otras estructuras social", lo que se relaciona, para Grootaert, con los niveles micro, meso y macro de la sociedad; razón por la cual implementa la metodología que incluye variables macroeconómicas, encuestas de hogares y aspectos institucionales y de gobierno.

Novakovsky (2003) en Jaramillo \& Szauer (2003), analiza el capital social de acuerdo a cinco características: a) es intangible; b) involucra aspectos subjetivos, culturales y de valores; c) produce beneficios para el individuo y la sociedad; d) es un bien público; y e) se incrementa con el uso. Partiendo de ello, Novakovsky tiene en cuenta un conjunto conceptos en respuesta de la dificultad que genera medir el capital social, es por ello, que implementa varias técnicas metodologías como encuestas de hogares, de opinión, indicadores y variables proxies.

Por su parte, Rojas \& Marin (2006) retoman la conceptualización de Putnam y Fukuyama, que consideran el capital social como una cooperación entre individuos que activan una confianza social con base a las normas y la cultura de la red de relaciones; y además establece una productividad que si no existiera el capital social no se generaría, tomando como referencia a Coleman. Es por ello que su metodología, la cual busca aproximarse a medir la confianza en este caso en una institución de educación, toma como referencia dichos autores.

Para Serrano, Alarcón \& Tassara (2006), por medio de una encuesta buscan recolectar la información necesaria para crear el índice de medición de capital social, basados en el concepto de Putnam y Coleman, analizando el capital social como el conglomerado de relaciones sociales y cooperaciones de los individuos basadas en la confianza para obtener beneficios. 
Michell \& Bosset (2007) basan su metodología de investigación principalmente en el Cuestionario de Capital Social Integrado diferenciando dos componentes del capital social, tomando en cuenta la concepción del capital social de Putnam y Coleman, los cuales diferencian dos partes: el lado estructural y cognitivo del mismo. Donde el primero, se basa en el comportamiento de las conexiones de red, mientras que el segundo, evidencia las actitudes de confianza y normativas de la sociedad.

Como se mencionó en líneas anteriores, los conceptos de participación y confianza, son claves para el capital social, el cual Valdivieso (2007) los relaciona teniendo en cuenta la participación en asociaciones políticas y colectivas (protestas, huelgas), que a su vez refuerza con Putnam. Asimismo, Sabatini (2009), en homologación con Valdivieso, tiene en cuenta una serie de indicadores para determinar la multidimensionalidad de la concepción de capital social, dado de que, al basarse en Coleman, el capital social se incorpora a varios contextos como culturales, institucionales, de normas y redes de relaciones interpersonales, necesidad por la cual el autor tiene en cuenta dicha metodología.

En la misma línea, aparece Rangel J. \& Saíz V. (2011) los cuales se basan en la concepción teórica de Putnam dado de que este le da mayor importancia a la confianza y cooperación, aspectos que se tienen en cuenta para la construcción del Índice de Gestión Social de los investigadores. A su vez, otros autores que se basan en Putnam y Coleman son: Piñas, Castellanos \& Morales (2011), Hurtado, García \& Copete (2012), Portela \& Neira (2012), dado de que la muestra de estudio busca obtener algún beneficio de sus redes de relaciones sociales, ya sea para el bienestar individual o general.

Finalmente, dada la diversidad de puntos de vista a lo largo de historia sobre la conceptualización del capital social, ya sea desde el aspecto sociológico o económico, hasta el momento no hay un declaración definitiva sobre el mismo, ya que a medida que van surgiendo investigaciones teorías y resultados al respecto, el concepto y sus metodologías van mutando junto a ellas. Es por ello que existen diferentes conceptualizaciones como metodologías.

El capital social ha sido un concepto que se ha ido fortaleciendo como se puede evidenciar en este documento de investigación, pero que todavía no hay un acuerdo generalizado sobre el alcance teórico y la medición del mismo. Más que ver la relación ente el concepto y la metodología, estos están relacionados en la forma en que se basa cada autor (sea la línea de Bourdieu, Putnam, Fukuyama o Coleman, entre otros) para determinar el método para recolectar la información del grupo de interés, y posteriormente aplicar el mecanismo de análisis sea por un modelo econométrico, análisis factorial, entre otros.

\section{CONCLUSIONES}

Como se evidenció en los apartados anteriores, hay una gran diversidad de propuestas metodológicas para aproximarse a una medición del capital social, desde encuestas por grupos independientes hasta encuestas realizadas por entidades a nivel mundial como la OCDE, el Banco Mundial, y oficinas de estadísticas de cada país, que posteriormente por medio de análisis descriptivos hacen un diagnóstico del capital social y se toman decisiones con respecto a ello. A su vez, por medio de esas encuestas también hacen estudios de caso, modelos econométricos, modelos de redes, análisis factorial, entre otros. Las anteriores metodologías se aplican a distintos grupos de interés como: países, ciudades, barrios, instituciones, grupo de estudiantes, empresas, es decir, en cualquier 
ente donde exista presencia de varios individuos que se relacionen entre sí para cumplir un objetivo en común. Todo lo anterior tenido en cuenta como elementos que influyen en la elección de una metodología.

Ahora bien, algunas ventajas y desventajas de las metodologías y herramientas implementadas por los autores en las investigaciones mencionadas se encuentran (su división no implica que hayan usado únicamente la herramienta metodológica en la cual se enmarca):

- Metodología de Evaluación Rápida del Capital Social (MERCS), aplicada por Durston (2001), que permite la organización y análisis práctico de la información sobre el capital social, pero que presenta un inconveniente, la ausencia del proceso para transformar ese análisis en evaluación. Es por ello que el autor recurre a la teoría fundamentada o grounded theory.

- La teoría fundamentada o grounded theory, aplicada por Durston (2001), Strauss y Corbin (2002) y González Ordoñez (2011), permite determinar la evaluación del capital social de manera estructurada e inductiva, basadas en hipótesis cercanas a la realidad social. Cabe resaltar que, dentro de la metodología, abarca etapas de recolección de información por medio de observación y entrevistas.

- Método de análisis cualitativo, el cual, por medio de encuestas, entrevistas, casos de estudio, interacciones con los grupos sociales e individuos, permiten la recolección y posterior interpretación por medio métodos cuantitativos como los modelos econométricos, codificaciones abiertas, axial o selectiva.

- Método de análisis cuantitativo por medio de modelos econométricos, utilizados por Grootaert (2001), Valdieso (20017), Pérez García, Serrano Martínez \& Fernández de Guevara Radoselovics (2008), Portela \& Neira (2012), Acquahh, Amoako-Gyampah \& Nyathi (2014) permite estimar el impacto que tiene el capital social, teniendo en cuenta aspectos cualitativos de los hogares o grupo de interés en particular; y distintas variables económicas, sociales, y de acuerdo al objetivo de la investigación de cada autor; igualmente se resalta la dificultad a la hora de la recolección de datos dados los sesgos en la información. A su vez, algunos autores como Palofox et. Al. (2013), Chung, Choi \& Seung-yoon (2014), y Fernandez Niño, Pinzón Flórez, Moreno Montoya \& Idrono Velanda (2014) utilizaron técnicas con modelos estructurales, modelos de maximoverosimulitud análisis factorial (implementado principalmente para la comprensión dimensional del capital social), estadísticos, y validación psicométrica.

- Como lo menciona Arraigada (2003), el capital social contiene en su mayoría aspectos cualitativos que necesita medir las acciones colectivas y la capacidad de resiliencia a situaciones adversas. Es por ello que los aspectos cualitativos tienen una mayor ventaja sobre los cuantitativos por medio de la investigación acción participativa dado de que permiten entender e interpretar de mejor manera los aspectos sociales de los grupos en una red de relaciones sociales.

- Las encuestas son una buena forma de medir el capital social (y la de mayor uso tanto en estudios cuantitativos como cualitativos), ya sea creada por los investigadores o los cuestionarios ya establecidos internacionalmente como el Cuestionario de Capital Social Integrado, dado de que es el principal medio 
de la recolección de información directa del grupo de interés, sean personas naturales o jurídicas, permitiendo conocer la variable confianza (que como se evidenció en el cuerpo del documento, es la variable proxie a capital social más usada) mediante rubros generales como la participación cívico-política, participaciones voluntarias, confianza en las relaciones sociales, entre otros aspectos mencionados con anterioridad. Entre sus debilidades se evidencia los sesgos en la información, la poca continuidad en la toma de información que permita tener un registro temporal, poca información sobre los grupos de asociaciones debidamente identificados, entre otros. Algunos autores que utilizan encuestas son: Novakovsky (2003), Ayerbe Eceberría et al. (2005), Rojas \& Martin (2006), Cheung \& Kwo-Hong (2010), Alcaide Lozano (2013), Palofox et al. (2013), Zambrano, Duque \& Manzano (2015)

- Por otro lado, se encuentras los autores que crearon un índice o conjunto de índices como otra metodología única o agregada, para medir el capital social, como Serrano, Alarcón \& Tassara (2006), Michell \& Bosset (2007), Sabatini (2009), Fornoni, Arribas \& Vila (2011), Rangel J. \& Saíz V. (2011), Piña, Castellanos \& Morales (2011), Hurtado, García \& Copete (2012), Portales Luis (2014), Saz Gil \& Gómez Quintero (2015) pero que para la construcción de los mismos, se necesitó el diseño e implementación de un cuestionario y entrevistas; igualmente se hizo necesario el uso de herramientas estadísticas de análisis como regresiones y análisis factorial.

Como se puede evidenciar, los autores no utilizan una sola herramienta metodológica para analizar y aproximarse a la medición del capital social, dada su complejidad y distintas concepciones, es por ello la implementación de instrumentos cualitativos y cuantitativos de manera conjunta, siendo indispensables en algunos casos, el uso de ambos.

En la actualidad no hay una metodología estándar para medir el capital social, es así como todavía se está en la búsqueda de cuál es la mejor forma de hacerlo, dado la complejidad y las múltiples dimensiones del mismo. Razón por la cual muchas investigaciones hablan de "una aproximación a la medición de capital", "un intento de medición de capital", etc.

A pesar de que existen diversas metodologías para medir el capital, las variables proxies o indicadores del mismo se mantienen, sea: confianza, pertenencia a un grupo u organización, participación ciudadana, entre otros. Asimismo, dado de que el concepto de capital social se ha ido expandiendo a medida que se van evidenciando hallazgos en las investigaciones, sus metodologías también van variando. El capital social y su medición, es un tema hoy por hoy en desarrollo que al tener diversos campos de acción y al estar involucrado el comportamiento humano en la composición y creación de redes de relaciones, es tan complejo y multidimensional que su análisis tanto cuantitativo como cualitativo es difícil de establecer de una manera general.

\section{REFERENCIAS BIBLIOGRÁFICAS}

Acquaah, M., Amoako-Gyampah, K., \& Nyathi, N. Q. (2014). Measurign and valuing social capital. Sout Africa: Network for Business Sustainability.

Alcaide Lozano, V. (2013). El capial social y las trayectorias laborales. Las redes personaes como mecanismos y recursos para la inserción y la trayectoria laboral. Barcelona: Tesis doctoral. Universidad Autónoma de Barcelona. 
Arriagada, I. (2003). Capital social: potencialidades y limitaciones analíticas de un concepto. CEPAL. SERIE Seminarios y conferencia $\mathrm{N}^{\circ} 31$.

Ayerbe Echeberría, M., Ayerbe Mujika, O., Barandiaran Irastorza, X., Lazkano Agirre, A., Mujika Alberdi, A., \& Plazaola Arrondo, A. (2005). El capital social de las organizaciones y su entorno: conceptualización teórica, medición e intervención en la generación del capital social. Ekonomiaz, 59, 14-47.

Batjargal, B. (2003). Social capital and entreprencurial performance in Russia: a longitudinal study. Organization Studies, 24, 535-556.

Bordieu, P. (2001). Poder, derecho y clases sociales (Segunda ed.). Bilbao: Editorial Desclée de Brouwe, S.A.

Bourdieu, P. (1985). Handbook of Theory and Research for the Sociology of Education. N.Y., Greenwood: J. Richardson.

Brown, L. D., \& Ashman, D. (1996). Participation, social capital, and intersectoral problem solving: african and asian cases. World Development, 24(9), 1467-1479.

Buciega, A., \& Esparcia, J. (2013). Desarrollo, territorio y capital social: Un análisis a partir de dinámicas relacionales en el desarrollo rural. REDES - Revista hispana para el análisis de redes sociales, 24(1), 81-113.

Burt, R. S. (1992). Structural holes: The social construction of competition. Cambridge: Harvard University Press.

Burt, R. S. (2000). The network structure of social capital. En R. I. Sutton, \& B. M. Staw, Research in organizational behavior (p.p. 345-423). New York: JAI Press.

Burt, R. S. (2001). Structural holes versus network closure as social capital. En N. Lin, K. Cook, \& R. S.

Burt, Social capital: Theory and research (p.p. 31-56). Hawthorne: Aldine de Gruyter.

Chaskin, R. J., Goerge, R. M., Skyles, A., \& Guiltinan, S. (2006). Measuring social capital: An exploration in community-research partnership. Journal of community Psychology, 34(4), 489-514.

Cheung, C.-K., \& Kwok-hong Chan, R. (2010). Social capital as exchange: Its contribution to morale. Social Indicators Research, 96(2), 205-227.

Chung, S., Choi, H., \& Seung-yoon Lee, S. (2014). Measuring social capital in the Republic of Korea with mixed methods: Application of factor analysis adn fuzzy-set ideal type approach. Social Indicators Research, 117(1), 45-64.

Coleman, J. S. (1988). Social Capital in the Creation of Human Capital. The American Journal of Sociology, 94, 95-120.

Coleman, J. S. (1990). Foundation of Social Theory. Cambridge: Harvard University Press. Recuperado el 23 de Febrero de 2016, de https://books.google.com.co/ books?hl=es\&lr=\&id=a4Dl8tiX4b8C\&oi=fnd\&pg=PR15\&dq=Coleman,+James.+1990.+ Foundation+of+Social+Theory,+Cambridge,+Harvard+University+Press.\&ots=qA3x Q2MWIo\&sig=wI2yytpQE3kPRakNVusD08W5bw4\#v=onepage\&q\&f=false

Durston, J. (2001). Evaluando capital social en comunidades campesinas en Chile. Ponencia preparada para presentación en el XXIII Congreso de LASA 2001. Washington, DC. 
Fernández Niño, J. A., Pinzón Flórez, C. E., Moreno Montoya, J., Cepeda Gil, M. C., \& Idrovo Velandia, Á. J. (2014). Capital social en áreas rurales: adapatación al español y validación factorial de una escala. Ciência \& Saúde Coletiva, 19(7), 2207-2214.

Fornoni, M., Arribas, I., \& Vila, J. E. (2011). Measurement of an individual entrepreneur's social capital: a multidimensional model. International Entrepreneurship and management Journal, 40, 495-507.

Fox, J. (1994). The difficult transition from clientelism to citizenship: lessons from Mexico. Wolrd politics, 46(2), 151-184.

Fukuyama, F. (1999). Social Capital and Civil Society, George Mason University. Obtenido de International Monetary Fund: https://www.imf.org/external/pubs/ft/seminar/1999/ reforms/fukuyama.htm\#V

Fukuyama, F. (2000). La gran ruptura. Naturaleza humana y reconstrucción del orden social. Ediciones B.

Gadermann, A. M., Zumbo, B., \& Guhn, M. (2012). Estimating ordinal reliability for Liketrt-type and ordinal item response data: A conceptual, empirical, and practical guide. Practical assessment, reseach \& evaluation, 17(3).

Gittel, R., \& Vidal, A. (1998). Community organizing: Building social capital as a development strategy. Thousand Oaks: Sage.

Glaeser, E. L., Laibson, D., \& Sacerdote, B. (2002). An economic approach to social capital. The Economic Journal, 112, 437-458.

González Ordóñez, A. (2011). Capital social en la comunidad ubicada en el sector Guayapa, parroquia Curimagua, municipio Petit, estado Falcón. Multiciencias, 11(2), 177-182.

Granovetter, M. S. (1973). The Strength of Weak Ties. (U. o. Press, Ed.) American Journal of Sociology, 78(6), 1360-1380.

Grootaert, C. (2001). Does social capital help the poor? A synthesis of findings from the local level institutions studies in Bolivia, Burkina Faso and Indonesia. Washington, DC: The World Bank. Local Level Institutions Working Paper No. 10.

Gulati, R. (1995). Social structure and alliance formation patterns: a longitudinal analysis. Administrative Science Quaterly, 40, 619-652.

Hall, P. A. (1999). Social Capital in Britain. British Journal of Political Science, 29(3), 417-461.

Hiernaux Espinosa, L. (2013). Densifircar nuestra vida colectiva: una propuesta de indicadores de capital social para Chile (Tesis de pregrado). Santiago de Chile: Universidad de Chile. Facultad de Economía y Negocios.

Hurtado, D., García, D., \& Copete, A. (2012). Tercera Medición del Capital Social en Colombia BARCAS 2011 - Medición de capital social en Cartagena. Bogotá: Fundación Antonio Restrepo Barco.

Jaramillo , F., \& Szauer, M. T. (2003). Capital social, vale para una agenda integral de desarrollo. Caracas: CAF.

Koka, B. R., \& Prescott, J. E. (2002). Strategic alliances as social capital: a multidimensional view. Strategic Management Journal, 1(23), 795-816. 
Laursen, K., Masciarelli, F., \& Prencipe, A. (2007). Regions matter: how regional characteristics affect external knowledge acquisition and innovation. Danish Research Unit for Industrial Dynamics (DRUID) Workin Paper No. 07-20.

Madrid de Pieters, N. (2003). Mecanismos de generación de capital social en programas gubernamuntales de desarrollo en Venezuela. En V. autore, Capital social. Clave para una agenda integral de desarrollo (p.p. 203-231). Venezuela: CAF.

Mitchell, A. D., \& Bossert, T. J. (2007). Measuring dimensions of social capital: Evidence from surveys in poor communities in Nicaragua. Social Science \& Medicine, 64, 50-63.

Nahapiet, J., \& Ghoshal, S. (1998). Social capital, intellectual capital, and the organizational advantage. The Academy of Management Review, 23(2), 242-266.

Narayan, D., \& Cassidy, M. F. (2001). A dimensional approach to measuring social capital: Development and validation of social capital inventory. Current Sociology, 49(2), 59-102.

Narayan, D., \& Pritchett, L. (1999). Cents and Socaibility: Household Income and Social Capital in Rural Tanzania. Chicago Journals. Economic Development and Cultural Change, 47(4), 871-897.

Novakovsky, I. (2003). Capital social y ética aplicada en proyectos de desarrollo. En A. varios, Capital Social, calve para una agenda integral de desarrollo (p.p. 177-202). Caracas, Venezuela: Dirección de Desarrollo Sostenible de la CAF.

OCDE. (2001). Measuring capital. A manual on the Measurement of Capital Stocks, Consumption of Fixed Capital and Capital Services. París: OCDE.

Palafox Moyers, C. G., Espejel Blanco, J. E., Burgos Flores, B., \& García Pequeño, J. A. (2013). El capital social como elemento de desarrollo en el municipio de Navojoa, Sonora, México. International Review of Business Research Papers, 9(2), 12-31.

Parnanen, A., \& Iisakka, L. (2006). Social Capital in Finland - Statistical Review. Statistics Finland. Prima Oy, Helsinki - Helsingfors.

Pérez García, F., Serrano Martínez, L., \& Fernández de Guevara Radoselovics, J. (2008). Estimación del capital social en España. España: Fundación BBVA.

Pérez García, F., Serrano Martínez, L., Montesinos Santalucía, V., \& Fernández de Guevara Radoselovics, J. (2006). Measurement of social capital and growth: An economic methodology. Bilbao: Documentos de trabajo 4. Fundación BBVA.

Piña Zambrano, H., Castellanos Tua, J., \& Morales Espinoza, A. (2011). Capital social en la cadena aloe, estado Falcón, Venezuela. Cuadernos de desarrollo rural, 8(66), 103-122.

Portales, L. (2014). Capital social y pobreza multidimensional, el caso de hogares pobres en Monterrey, México. Convergencia. Revista de Ciencias Sociales, 21(66), 39-63.

Portela, M., \& Neira, I. (2012). El papel del capital social en la ayuda al desarrollo: Un primer análisis para la OCDE. Revista de Economía Mundial(30), 185-208.

Portes, A. (1998). Social Capital: Its Origins and Applications in Modern Sociology. Annual Review of Sociology, 24, 1-24.

Putnam, R. D. (1995). Bowling alone: America's declinin social capital. Journal of Democracy, 6(1), 65-78.

Putnam, R. D. (2001). Gesellschaft und Gemeinsinn. Bertelsmann Foundation Publishers. 
Putnam, R. D., Leonardi, R., \& Nanetti, R. (1993). Making Democracy Work: Civic Traditions in Modern Italy. New Jersey: Princeton University Press.

Rangel J., S. A., \& Saíz V., J. E. (2011). Índice de Gestión Social Empresarial (IGS): una aplicación de la medición de capital social. Revista CIFE, 17(12), 73-101.

Rojas Lopez, M. D., \& Marin, S. P. (2006). Aproximaciones a la medición de la confianza. Dyna, 73(150), 119-130.

Sabatini, F. (2009). Social capital as social networks: a new framework for measurement and an empirical analysis of its determinants and consequences. The Jounal of Socio-Economics, $38,429-442$.

Saegert, S., Thompson, J. P., \& Warren, M. R. (2001). Social capital and poor communities. New York: Russell Sage Foundation.

Sampson, R. J. (1999). What "community supplies. En R. Ferguson, \& T. Dickens, Urban problems and community developments (p.p. 241-292). Washington, DC: The Brookings Institution Press.

Saz Gil, M. I., \& Gómez Quintero, J. D. (2015). Una aproximación a la cuantificación y caracterización del capital social: una variable relevante en el desarrollo de la provincia de Teruel, España. EURE, 41(123), 29-51.

Serrano, C., Alarcón, A., \& Tassara, G. (2006). Diseño y aplicación de índice integrado de capital social en tres barrios urbanos de la región de Coquimbo. Programa Más Región: Asesorías para el Desarrollo S.A.

Strauss, A., \& Corbin, J. (2002). Bases de la investigación cualitativa. Técnicas y procedimientos para desarrollar la teoría fundamentada. Medellín: Editorial Universidad de Antioquia.

Sudarsky, J. R. (2007). La evolución del capital social en Colombia, 1997-2005. Bogotá: Fundación Antonio Restrepo Barco.

Tendler, J., \& Freedheim, S. (1994). Trust in a rent-seeking world: Health and government transformed in Northeast Brazil. World Development, 22(12), 1771-1792.

Universidad del Pacífico. (2009). Capital Social: Conceptualizción, enfoques y medicones. Ecuador.

Valdivieso, P. (2007). Capital social en Chile - mediciones y especificaciones. Revista Política, 48, 53-75.

Van Der Gaag, M., \& Snijders, T. A. (2003). A comparison of measures for individual social capital. ICS. University of Groningen. Vrije Universiteit Amsterdam.

Van Der Gaag, M., \& Snijders, T. A. (2003). The resource generator: social capital quantification with concrete items. ICS. University of Groningen.

Wang, H., Schlesinger, M., Wang, H., \& Hsiao, W. (2009). The flip-side of social capital: The distinctive influences of trust and mistrust on health in rural China. Social Science \& Medicine, 68 , 133-142.

Warren, M. R. (2001). Dry bones rattling: Community building to revitalize American democracy. Princeton: Princeton University Press.

Woolcock, M. (1998). Social capital and economic development: Toward a theoretical synthesis and policy framework. Theory and Society, 27, 151-208. 
Woolcock, M. (2001). The place of social capital in understanding social and economics outcomes. Canadian Journal of Policy Research, 2(1).

Zambrano Miranda, M., Duque Sastre, J. E., \& Manzano, J. (2015). Medición del capital social: un análisis aplicado al barrio La Primavera. Cúcuta: Observatorio Socioeconómico Regional de la Frontera.

Zarazúa, J. A., Almaguer Vargas, G., \& Rendón Medel, R. (2012). Capital social. Casdo red de innovación de maíz en Zamora, Michoacán, México. Cuadernos de desarrollo rural, 9(68), 105-124. 